\title{
Does point-of-care ultrasonography (POCUS) reduce clinical failure rates in patients undergoing incision and drainage for an uncomplicated abscess?
}

Ian Surdhar, MD, MSc*; Anne Finlayson, MD*; Tomislav Jelic, MD*

\author{
Abstract link: https://doi.org/10.1016/j.annemergmed. \\ 2018.05.014 \\ Full citation: Gaspari RJ, Sanseverino A, Gleeson \\ $T$. Abscess incision and drainage with or without \\ ultrasonography: a randomized controlled trial. \\ Ann Emerg Med 2019;73:1-7. \\ Article type: Therapy \\ Ratings: Methods - 2.5/5 Usefulness - 3.5/5
}

\section{INTRODUCTION}

\section{Background}

There is limited evidence to suggest that point-of-care ultrasonography (POCUS) improves outcomes in the emergency department (ED) management of patients with a suspected abscess.

\section{Objective}

The aim of this study was to compare clinical failure rates of abscess drainage in patients who underwent ED incision and drainage with and without POCUS.

\section{METHODS}

\section{Design}

Randomized controlled trial

\section{Setting}

Large academic ED

\section{Subjects}

Subjects included adult patients with a suspected uncomplicated abscess. Exclusion criteria included "ill appearing patients" and patients with abscesses related to animal bites or foreign bodies, paronychia, dental, genital, or peritonsillar abscesses.

\section{Intervention}

Incision and drainage were guided by physical exam (PE) and POCUS versus PE alone. All assessments were performed by residents or attending physicians with experience in soft tissue POCUS.

\section{Outcomes}

The primary outcome was failure of therapy defined by repeated incision and drainage, which produced purulent drainage. Secondary outcomes included the need for additional antibiotics, as well as continued pain and purulent discharge at follow-up.

\section{RESULTS}

Patients totalling 452 presented to the study ED during the study period. Of these, 125 patients were enrolled: 63 to the PE and POCUS group, and 62 to the PE-alone group. After misallocation and loss to follow-up, 54 patients remained in the PE and POCUS group and 53 in the PE-alone group. There was no difference in baseline characteristics between groups with respect to abscess size, duration of symptoms, percentage with

From the *Department of Emergency Medicine, University of Manitoba, Winnipeg, MB.

Correspondence to: Dr. Tomislav Jelic, Department of Emergency Medicine, Max Rady College of Medicine Rady Faculty of Health Sciences, University of Manitoba, S203 Medical Services Building 750 Bannatyne Ave., Winnipeg, MB R3E 0W2; Email: tjelic@gmail.com.

(c) Canadian Association of Emergency Physicians

CJEM 2019;21(4):552-553

DOI 10.1017/cem.2019.32 
cellulitis, and treatment with antibiotics. The overall failure rate was $10.3 \%$ (95\% confidence interval [CI] 5.717.6). PE and POCUS patients were less likely to fail therapy $(3.7 \%$ v. $17.0 \%)$ with a between-groups difference of $13.3 \%$ (95\% CI 0.0-19.4\%). Multivariate logistic modelling identified the largest predictor of failure to be lack of ultrasonography.

\section{APPRAISAL}

\section{Strengths}

- Common ED complaint

- First study to evaluate effectiveness for POCUS in treating abscesses

- Strong randomized design

- Well-described methods

- Good efforts to blind outcome assessments, although this may not always have succeeded

- Detailed data analyses

\section{Limitations}

- A major weakness is failure to make the intention to treat the primary analysis. This was given as a secondary analysis and found no differences between groups. The per protocol analysis is of much less importance because it excluded patients who crossed over or were lost to follow-up.

- A second concern is that the primary outcome measure "requiring a second I\&D" was at the discretion of the physician, and the decision for doing so was not explicitly described. More patient-oriented outcomes such as relief of pain at Day 10 could have been considered primary and would have allowed more complete follow-up. As it was, the secondary outcomes were not different between groups.

- Most patients in the PE group did not have a diagnosis based upon POCUS, and this may have led to selection bias.

- The sample size was not based upon an a priori estimate of the minimal clinically importance difference; given this, the failure rate was very low in the POCUS group.
- Single site study that should be repeated at other EDs.

- Small differences between the study groups, although adjusted for by multivariate analysis.

\section{CONTEXT}

Multiple studies suggest that POCUS helps differentiate abscess from cellulitis in patients presenting to the ED with skin and soft tissue infections. ${ }^{1}$ POCUS has further been shown to be more sensitive but less specific than CT in identifying superficial abscesses. ${ }^{2}$ This is the first prospective randomized clinical trial to examine the impact of ultrasonography on rates of clinical failure in abscess drainage. A larger trial confirming the results of this study would be helpful and could also involve an examination of other outcomes such as rates of accidental incision and drainage of non-abscesses when using POCUS versus $\mathrm{PE}$ alone.

\section{BOTTOM LINE}

Skin and soft tissue infections are a common presenting complaint in the ED, and treatment failure is common. This was the first trial to evaluate the effectiveness of POCUS for treating cutaneous abscesses. POCUS may reduce the failure rate of incision and drainage of abscesses in the ED but further studies would be helpful.

Keywords: Journal Club, POCUS, skin and soft tissue ultrasound

Competing interests: None declared.

\section{REFERENCES}

1. Barbic D, Chenkin J, Cho DD, et al. In patients presenting to the emergency department with skin and soft tissue infections what is the diagnostic accuracy of point-of-care ultrasonography for the diagnosis of abscess compared to the current standard of care? A systematic review and meta-analysis. BM7 Open 2017;7:e013688.

2. Gaspari R, Dayno M, Briones J, Blehar D. Comparison of computerized tomography and ultrasound for diagnosing soft tissue abscesses. Crit Ultrasound 7 2012;4(1):5. 